\title{
Production Inventories Policies for Three Levels of Production with Stock-Dependent Demand and Inflation
}

\author{
Jayshree Kathuria $^{\# 1}$, S.R. Singh ${ }^{\# 2}$, \\ Department of Mathematics, C.C.S. University, Meerut -250004, INDIA \\ ${ }^{1}$ Jayashri000@gmail.com \\ Department of Mathematics, C.C.S. University, Meerut-250004, INDIA \\ ${ }^{2}$ shivrajpundir@gmail.com
}

\begin{abstract}
The managing of inventories is one of the next significant tasks, in order to find out this concept the manager have tried to do in that appropriate way through which the cost associated with inventory can reduce and profit may grow, with the advent of better technologies if manufacturing and retail industry, an EPQ model plays an important role in production and manufacturing system. It is evident from the forgoing, that demand structure occupies central position in inventory modelling. Researchers have usually observed that the inventory and sales are not independent of one another. An increase in inventories may bring about increased sales of some items. Furthermore in the unstable global economic structure, inflation plays a vital role before making any investment. So, this article develops three different levels of production rates with stock related demand for deteriorating items under inflationary condition. The total cost of production is dependent on production rate demand rate and rate of deteriorating items. It has assumed production started at one rate and switch over to another after a certain time lag, thus for low production rate in starting leading to reduction in holding cost to stock the large quantity of items is ignored. Our focus in this study is on determining the optimal solution of production time so that total cost of the whole cycle will be minimized.
\end{abstract}

Keyword - EPQ1, deterioration2, inflation3, three levels of production4, stock-dependent demand5

\section{INTRODUCTION}

As long arm of grinding, deep financial crisis continues to haunt the wobbling economy since the recent and continued surge in the inflation rate, connects and hypotheses about its many economic effects literally means to blow up or get bigger. As it has been seen that the different inflation rates in the volatile market structure do not just have an effect on the purchasing lot but they also stimulate the demand for products. Further from the financial perspective get the real estimate of all costs incurred in financial decision making inflation plays a crucial role while determining the optimal inventory policy. To relax the assumption of no inflationary effects on costs, Buzacott (1975) and Misra (1975) were the first whom developed on EOQ model with a constant inflation rate. Recently, a large number of inventory models dealing with inflation like Jayshree \& Jain (2016), Jayshree \& Singh (2016 a).

The present paper establishes EPQ framework with the combined effect of inflation, deterioration. Now, items kept as inventory are tacitly assumed to have an infinite life span but items like fruits and vegetables and medicines have a shelf life and begin to deteriorate after a time this phenomenon is termed as deterioration. Thus, another phenomenon that significantly affects items in inventory e.g. chemicals, food items gasoline, medicine, drugs which deteriorate with time, is deterioration. Several research papers in this interesting area were published by researchers in the last few decades such as Raafat et al. (1991), Goyal and Giri (2001), Jayshree and Singh (2016 b). With the advent of technologies in production and manufacturing structure, it is possible that the production is started at one rate and after that switches to another. It may yield to lead to reduction in holding cost, a large amount of stock of manufactured items at the starting stage is often ignored. Hsu and Hsu (2013) suggested an integrated model where the vendor's production process is imperfect and planned back orders. Sivasankari and Panayappan (2014) visited the EPQ model for two level of production with deterioration. Sarkar et al. (2014) production model with random defective rate and rework process. Except these a good number of works have been published in this area viz; Barron et al (2014a, 2014b), Taleizadeh et al. (2015). Recently Krishnamoorthi \& Sivasankari (2016) studied a production model with three levels of production and shortages. Now, more recently the work of Viji \& karthikeyan (in press) to propose and EPQ model with weibull deterioration and shortages.

In classical inventory models, the demand rate of items was often considered either large amount of constant or time dependent. Either large amount of inventory on display researchers observed that the demand rate of many items usually influenced. In supermarket concept, to display the large piles of goods will lead customers to buy more. Customers may have the opportunity to choose wide range of items based on the 
observed phenomenon the consumption rate may be influenced by stock levels to reflect sales in different phases of product life cycles which depends on the actual rate of withdrawal of items from the inventory.

Firstly inventory model for stock-dependent consumption rate was established by Gupta and Vrat (1986). Giri et al (1996) proposed a model with deterioration and considered demand function Baker and Urban (1988) developed a deterministic inventory system in which demand of item is a polynomial function of the instantaneous stock level. Moreover $\mathrm{Wu}$ et al (2006) studied an optimal replenishment policy with stock dependent demand and shortages. Furthermore many extensive models were established by researchers such as Levin (1975) Dye and Ouyang (2005), Alfares (2007) etc; their work is focused on the considered assumption.

Keeping up with the above scenario, a model has been developed under inflation and deterioration in which three different levels of production are considered. It has been assumed the production started at one rate and after certain time length may be switch over to another rate. This is desirable situation we avoided to stock a large quantity of manufactured item initially due to the start at a low rate of production which reduces to holding cost. This model is developed without shortages. Mathematical model is developed to depict the above scenario. Finally a numerical example and sensitivity analysis also been presented.

\section{Assumptions \& Notations}

Assumptions: The assumptions of an inventory model are as follows:

The production rate is known and constant.

The demand rate is known and stock-dependent.

$\mathrm{D}=\mathrm{k}+\alpha \mathrm{Q}(\mathrm{t}) \quad$ where $\mathrm{k}, \alpha$ are non negative.

Items are produced and added to the inventory.

Three rates of production are considered.

The item is a single Product; it does not interact with any other inventory items.

The production rate is always greater than or equal to the sum of the demand rate.

The inventory system involves only one item and the lead time is considered to be negligible.

\section{Notations:}

$\mathrm{P}$ - Production rate in unit time,

$\theta$ - constant deterioration rate,

$\mathrm{Q}_{1}$ - on hand inventory level at time $\mathrm{T}_{1}$,

$\mathrm{Q}_{2}$ - on hand inventory level at time $\mathrm{T}_{2}$,

$\mathrm{Q}_{3}$ - on hand inventory level at time $\mathrm{T}_{3}$,

$\mathrm{Q}^{*}$ - the total production quantity,

$\mathrm{Cp}$ - Production Cost per unit,

$\mathrm{C}_{\mathrm{h}}$-Holding cost per unit/ per unit time,

$\mathrm{C}_{\mathrm{O}}$ - Setup cost per production cycle at $\mathrm{T}=0$,

$\mathrm{T}-$ length of the inventory cycle,

$T_{i}$ - unit time in periods where $i=(1,2,3)$,

TC - Total cost

\section{III.MATHEMATICAL MODELS}

\section{Production inventory model for three levels of production}

The changes in inventory level against time are represented in fig. 1 . The first production setup starts with zero inventory at $\mathrm{t}=0$ and end at $\mathrm{t}=\mathrm{T}$. During the duration $\left(0, \mathrm{~T}_{1}\right)$, the inventory level increases where demand less than production and with deterioration the maximum inventory level $\mathrm{Q}_{1}$ is reached at $\mathrm{t}=\mathrm{T}_{1}$.

\section{Inventory Level}

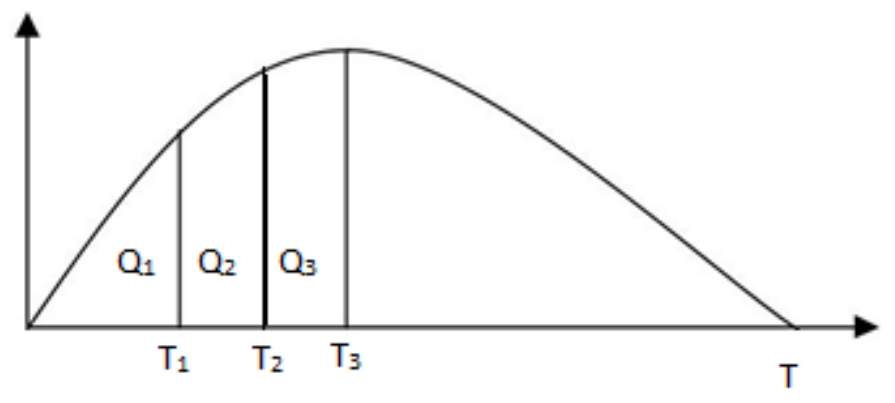

Fig 1 : On hand inventory of three levels of production with deteriorative items 
Therefore, the maximum inventory level equal to $(\mathrm{P}-\mathrm{k}) \mathrm{T}_{1}$. During time $\mathrm{T}_{2}$, Production increases at the rate of "a" time of P for P-D i.e. (aP-D) where " $a$ " is a constant. Therefore, the maximum inventory level equal to (a P-k) $T_{2}$. During time $T_{3}$, Production increases at the rate of " $b$ " time of ( b P-D)i.e. b P-D where " $b$ " is a constant. Therefore, the maximum inventory level equal to (b P-k) $T_{3}$. During decline time, the inventory level starts to decrease due to demand at a rate D up to time T. Here, D is considered stock dependent demand. Let $\mathrm{Q}(\mathrm{t})$ denote the inventory level of the system at time $\mathrm{T}$. The differential equations describing the system in the interval $(0, \mathrm{~T})$ given by

Let $\mathrm{Q}(\mathrm{t})$ assume the Inventory level of the system at time T. The differential equations describing the system in the time duration $(0, \mathrm{~T})$ are defined as

$$
\begin{array}{ll}
\mathrm{Q}^{\prime}(\mathrm{t})+\theta \mathrm{Q}(\mathrm{t})=\mathrm{P}-\mathrm{k}-\alpha \mathrm{Q}(\mathrm{t}) ; & 0 \leq \mathrm{t} \leq \mathrm{T}_{1} \\
\mathrm{Q}^{\prime}(\mathrm{t})+\theta \mathrm{Q}(\mathrm{t})=\mathrm{aP}-\mathrm{k}-\alpha \mathrm{Q}(\mathrm{t}) ; & \mathrm{T}_{1} \leq \mathrm{t} \leq \mathrm{T}_{2} \\
\mathrm{Q}^{\prime}(\mathrm{t})+\theta \mathrm{Q}(\mathrm{t})=\mathrm{bP}-\mathrm{k}-\alpha \mathrm{Q}(\mathrm{t}) ; & \mathrm{T}_{2} \leq \mathrm{t} \leq \mathrm{T}_{3} \\
\mathrm{Q}^{\prime}(\mathrm{t})+\theta \mathrm{Q}(\mathrm{t})=-\mathrm{k}-\alpha \mathrm{Q}(\mathrm{t}) ; & \mathrm{T}_{3} \leq \mathrm{t} \leq \mathrm{T}
\end{array}
$$

The boundary conditions are

$$
\mathrm{Q}(0)=0, \mathrm{Q}\left(\mathrm{T}_{1}\right)=\mathrm{Q}_{1}, \quad \mathrm{Q}\left(\mathrm{T}_{2}\right)=\mathrm{Q}_{2}, \quad \mathrm{Q}\left(\mathrm{T}_{3}\right)=\mathrm{Q}_{3}, \quad \mathrm{Q}(\mathrm{T})=0
$$

The solution of the differential equations are given as

$$
\begin{array}{ll}
\mathrm{Q}(\mathrm{t})=\frac{P-k}{\alpha+\theta}\left[1-e^{-(\alpha+\theta) t}\right] ; & 0 \leq \mathrm{t} \leq \mathrm{T}_{1} \\
\mathrm{Q}(\mathrm{t}) \frac{a P-k}{\alpha+\theta}\left[1-e^{-(\alpha+\theta) t}\right] ; & \mathrm{T}_{1} \leq \mathrm{t} \leq \mathrm{T}_{2} \\
\mathrm{Q}(\mathrm{t})=\frac{b P-k}{\alpha+\theta}\left[1-e^{-(\alpha+\theta) t}\right] ; & \mathrm{T}_{2} \leq \mathrm{t} \leq \mathrm{T}_{3} \\
\mathrm{Q}(\mathrm{t})=\frac{k}{\alpha+\theta}\left[e^{(\alpha+\theta)(T-t)}-1\right] ; & \mathrm{T}_{3} \leq \mathrm{t} \leq \mathrm{T}
\end{array}
$$

The maximum inventory level during time $T_{1}$ is calculated as follows. From equations (5) and (6),

$$
\mathrm{Q}_{1}=\frac{P-k}{\alpha+\theta}\left[1-e^{-(\alpha+\theta) T_{1}}\right]
$$

By expansion and neglecting the second and higher power of $\theta$ we have

Therefore,

$$
\mathrm{Q}_{1}=(\mathrm{P}-\mathrm{k}) \mathrm{T}_{1}
$$

The maximum inventory level $\mathrm{Q}_{2}$ in time duration $\mathrm{T}_{2}$ is calculated as follows

$$
\mathrm{Q}_{2}=(\mathrm{a} \mathrm{P}-\mathrm{k}) \mathrm{T}_{2}
$$

The maximum inventory level $\mathrm{Q}_{3}$ in time duration $\mathrm{T}_{3}$ is defined as follows by neglecting the second and higher power terms of $\theta$

$$
\mathrm{Q}_{3}=(\mathrm{bP}-\mathrm{k}) \mathrm{T}_{3}
$$

Total Cost: The sum of the production cost, ordering cost, holding cost and deteriorating cost is defined as the total cost. They are grouped together after evaluating the above cost individually.

i) Production cost $=C_{P}\left\{\mathrm{P}+\mathrm{a} \mathrm{P}\left(\mathrm{T}_{2}-\mathrm{T}_{1}\right)+\mathrm{b} \mathrm{P}\left(\mathrm{T}_{3}-\mathrm{T}_{2}\right)\right\}$

ii) Setup cost per set $=\frac{C o}{T}$

iii) Holding cost per unit time in inflationary environment

$$
\begin{gathered}
=\frac{C_{h}}{T}\left[\int_{0}^{T_{1}} e^{-r t} Q(t) d t+\int_{T_{1}}^{T_{2}} e^{-r t} Q(t) d t+\int_{T_{2}}^{T_{3}} e^{-r t} Q(t) d t+\int_{T_{3}}^{T_{4}} e^{-r t} Q(t) d t\right]=\frac{C_{h}}{T}\left[\int_{0}^{T_{1}} \frac{P-k}{(\alpha+\theta)}\{1-\right. \\
\left.e^{-(\alpha+\theta) t}\right\}(1-r t) d t+\int_{T_{1}}^{T_{2}} \frac{a P-k}{(\alpha+\theta)}\left\{1-e^{-(\alpha+\theta) t}\right\}(1-r t) d t+\int_{T_{2}}^{T_{3}} \frac{b P-k}{(\alpha+\theta)}\left\{1-e^{-(\alpha+\theta) t}\right\}(1-r t) d t+ \\
\left.\int_{T_{3}}^{T_{4}} \frac{k}{(\alpha+\theta)}\left\{e^{(\alpha+\theta)(T-t)}-1\right\}(1-r t) d t\right] \\
=\frac{C_{h}}{T}\left[P\left(\frac{t^{2}}{2}-\frac{r t^{3}}{3}\right)_{0}^{T_{1}}+(a P-k)\left(\frac{t^{2}}{2}-\frac{r t^{3}}{3}\right)_{T_{1}}^{T_{2}}+(b P-k)\left(\frac{t^{2}}{2}-\frac{r t^{3}}{3}\right)_{T_{2}}^{T_{3}}+k\left(T_{4} t-\frac{t^{2}}{2}-\frac{r T_{4} t^{2}}{2}+\right.\right. \\
\left.\left.\frac{r t^{3}}{3}\right)_{T_{3}}^{T_{4}}\right] \\
=\frac{C_{h}}{T}\left[(P-k)\left(\frac{T_{1}^{2}}{2}-\frac{r T_{1}{ }^{3}}{3}\right)+(a P-k)\left(\frac{T_{2}{ }^{2}}{2}-\frac{T_{1}{ }^{2}}{2}-\frac{r T_{2}{ }^{3}}{3}+\frac{r T_{1}{ }^{3}}{3}\right)+(b P-k)\left(\frac{T_{3}{ }^{2}}{2}-\frac{T_{2}{ }^{2}}{2}-\frac{r T_{3}{ }^{3}}{3}+\right.\right. \\
\left.\left.\frac{r T_{2}{ }^{3}}{3}\right)+k\left(\frac{T^{2}}{2}-T T_{3}+\frac{T_{3}{ }^{2}}{2}-\frac{r T^{3}}{6}-\frac{r T_{3}{ }^{3}}{6}+\frac{r T T_{3}{ }^{2}}{2}\right)\right]
\end{gathered}
$$

iv) Deterioration cost per unit time with inflation

$$
=\frac{C_{P}}{T}\left[\int_{0}^{T_{1}} e^{-r t} Q(t) d t+\int_{T_{1}}^{T_{2}} e^{-r t} Q(t) d t+\int_{T_{2}}^{T_{3}} e^{-r t} Q(t) d t+\int_{T_{3}}^{T_{4}} e^{-r t} Q(t) d t\right]
$$




$$
=\frac{C_{P}}{T}\left[(P-k)\left(\frac{T_{1}^{2}}{2}-\frac{r T_{1}{ }^{3}}{3}\right)+(a P-k)\left(\frac{T_{2}{ }^{2}}{2}-\frac{T_{1}{ }^{2}}{2}-\frac{r T_{2}{ }^{3}}{3}+\frac{r T_{1}{ }^{3}}{3}\right)+(b P-k)\left(\frac{T_{3}{ }^{2}}{2}-\frac{T_{2}{ }^{2}}{2}-\frac{r T_{3}{ }^{3}}{3}+\frac{r T_{2}{ }^{3}}{3}\right)+\right.
$$
$\left.k\left(\frac{T^{2}}{2}-T T_{3}+\frac{T_{3}{ }^{2}}{2}-\frac{r T^{3}}{6}-\frac{r T_{3}{ }^{3}}{6}+\frac{r T T_{3}{ }^{2}}{2}\right)\right]$

v) Total Cost $=$ Production Cost + Ordering Cost + Holding Cost + Deterioration Cost

$=C_{P}\left\{\mathrm{P} \mathrm{T}_{1}+\mathrm{a} \mathrm{P}\left(\mathrm{T}_{2}-\mathrm{T}_{1}\right)+\mathrm{b} \mathrm{P}\left(\mathrm{T}_{3}-\mathrm{T}_{2}\right)\right\}+\frac{C o}{T}+\frac{C_{h}}{T}\left[(P-k)\left(\frac{T_{1}^{2}}{2}-\frac{r T_{1}{ }^{3}}{3}\right)+(a P-k)\left(\frac{T_{2}{ }^{2}}{2}-\frac{T_{1}{ }^{2}}{2}-\frac{r T_{2}{ }^{3}}{3}+\frac{r T_{1}{ }^{3}}{3}\right)+\right.$ $\left.(b P-k)\left(\frac{T_{3}{ }^{2}}{2}-\frac{T_{2}{ }^{2}}{2}-\frac{r T_{3}{ }^{3}}{3}+\frac{r T_{2}{ }^{3}}{3}\right)+k\left(\frac{T^{2}}{2}-T T_{3}+\frac{T_{3}{ }^{2}}{2}-\frac{r T^{3}}{6}-\frac{r T_{3}{ }^{3}}{6}+\frac{r T T_{3}{ }^{2}}{2}\right)\right]+\frac{C_{P}}{T}\left[(P-k)\left(\frac{T_{1}^{2}}{2}-\frac{r T_{1}{ }^{3}}{3}\right)+\right.$ $(a P-k)\left(\frac{T_{2}{ }^{2}}{2}-\frac{T_{1}{ }^{2}}{2}-\frac{r T_{2}{ }^{3}}{3}+\frac{r T_{1}{ }^{3}}{3}\right)+(b P-k)\left(\frac{T_{3}{ }^{2}}{2}-\frac{T_{2}{ }^{2}}{2}-\frac{r T_{3}{ }^{3}}{3}+\frac{r T_{2}{ }^{3}}{3}\right)+k\left(\frac{T^{2}}{2}-T T_{3}+\frac{T_{3}{ }^{2}}{2}-\frac{r T^{3}}{6}-\frac{r T_{3}{ }^{3}}{6}+\right.$ $\left.\left.\frac{r T T_{3}^{2}}{2}\right)\right]$

Let $\mathrm{T}_{1=} \beta \mathrm{T}_{3}$ and $\mathrm{T}_{2}=\gamma \mathrm{T}_{3}$

Now, we get

Total Cost $=\left[C_{\mathrm{P}}\left\{\left(P \beta T_{3}+a P(Y-\beta) T_{3}+b P\left(T_{3}-Y T_{3}\right)\right\}+\frac{C_{0}}{T}+\frac{\left(C_{h}+\theta C_{\mathrm{P}}\right)}{T}\left\{(P-k)\left(\frac{\beta^{2} T_{3}^{2}}{2}-\frac{r \beta^{3} T_{3}^{3}}{3}\right)+\right.\right.\right.$ $(a P-k)\left(\left(Y^{2}-\beta^{2}\right) \frac{T_{3}{ }^{2}}{2}-\frac{r T_{3}{ }^{3}}{3}\left(Y^{3}-\beta^{3}\right)\right)+(b P-k)\left(\frac{T_{3}{ }^{2}}{2}\left(1-Y^{2}\right)-\frac{1}{3} r T_{3}{ }^{3}\left(1-\Upsilon^{3}\right)\right)+k\left(\frac{\mathrm{T}^{2}}{2}-T T_{3}+\right.$ $\left.\left.\left.\frac{T_{3}^{2}}{2}-\frac{r t^{3}}{6}-\frac{r T_{3}^{3}}{3}+\frac{r T_{3}^{2} \mathrm{~T}}{2}\right)\right\}\right]$

Partially differentiate the equation (19), w.r.t. $T_{3}$

$$
\begin{gathered}
(P \beta+b P(1-\gamma)+a P(-\beta+\Upsilon))+\frac{1}{\mathrm{~T}}\left(C_{\mathrm{P}} \theta+C_{h}\right)\left(k\left(-T+T_{3}+r T T_{3}-r T_{3}^{2}\right)+(-k+\right. \\
P)\left(\beta^{2} T_{3}-r \beta^{3} T_{3}^{2}\right)+(-k+b P)\left(\left(1-\gamma^{2}\right) T_{3}-r\left(1-\gamma^{3}\right) T_{3}^{2}\right)+(-k+a P)\left(\left(-\beta^{2}+\right.\right. \\
\left.\left.\left.\gamma^{2}\right) T_{3}-r\left(-\beta^{3}+\gamma^{3}\right) T_{3}^{2}\right)\right)=0 \\
\frac{\partial^{2}}{\partial \mathrm{T}_{3}^{2}}(\mathrm{~T} . \mathrm{C})=\frac{1}{T}\left(C_{\mathrm{P}} \theta+C_{h}\right)\left(k\left(1+r T-2 r T_{3}\right)+(-k+P)\left(\beta^{2}-2 r \beta^{3} T_{3}\right)+(-k+b P)\left(1-\Upsilon^{2}-\right.\right. \\
\left.\left.2 r\left(1-\gamma^{3}\right) T_{3}\right)+(-k+a P)\left(-\beta^{2}+\gamma^{2}-2 r\left(-\beta^{3}+\Upsilon^{3}\right) T_{3}\right)\right)>0
\end{gathered}
$$

Partially differentiate the equation (19) w.r.t. T

$$
\begin{aligned}
-\frac{C_{0}}{T^{2}}+\frac{k\left(C_{\mathrm{P}} \theta+C_{h}\right)\left(T-\frac{r T^{2}}{2}-T_{3}+\frac{r T_{3}^{2}}{2}\right)}{T} & \\
& -\frac{1}{T^{2}}\left(C_{\mathrm{P}} \theta+C_{h}\right)\left(k\left(\frac{T^{2}}{2}-\frac{r T^{3}}{6}-T T_{3}+\frac{T_{3}^{2}}{2}+\frac{1}{2} r T T_{3}^{2}-\frac{r T_{3}^{3}}{3}\right)\right. \\
& +(-k+P)\left(\frac{1}{2} \beta^{2} T_{3}^{2}-\frac{1}{3} r \beta^{3} T_{3}^{3}\right)+(-k+b P)\left(\frac{1}{2}\left(1-r^{2}\right) T_{3}^{2}-\frac{1}{3} r\left(1-r^{3}\right) T_{3}^{3}\right) \\
& \left.+(-k+a P)\left(\frac{1}{2}\left(-\beta^{2}+r^{2}\right) T_{3}^{2}-\frac{1}{3} r\left(-\beta^{3}+r^{3}\right) T_{3}^{3}\right)\right)=0
\end{aligned}
$$

Again partially differentiate the above equation w.r.t. T

$$
\begin{aligned}
\frac{2 C_{0}}{T^{3}}+\frac{k(1-r T)}{}\left(C_{\mathrm{P}} \theta+C_{h}\right) & -\frac{2 k\left(C_{\mathrm{P}} \theta+C_{h}\right)\left(T-\frac{r T^{2}}{2}-T_{3}+\frac{r T_{3}^{2}}{2}\right)}{T^{2}} \\
& +\frac{1}{T^{3}} 2\left(C_{P} \theta+C_{h}\right)\left(k\left(\frac{T^{2}}{2}-\frac{r T^{3}}{6}-T T_{3}+\frac{T_{3}^{2}}{2}+\frac{1}{2} r T T_{3}^{2}-\frac{r T_{3}^{3}}{3}\right)\right. \\
& +(-k+P)\left(\frac{1}{2} \beta^{2} T_{3}^{2}-\frac{1}{3} r \beta^{3} T_{3}^{3}\right)+(-k+b P)\left(\frac{1}{2}\left(1-r^{2}\right) T_{3}^{2}-\frac{1}{3} r\left(1-r^{3}\right) T_{3}^{3}\right) \\
& \left.+(-k+a P)\left(\frac{1}{2}\left(-\beta^{2}+r^{2}\right) T_{3}^{2}-\frac{1}{3} r\left(-\beta^{3}+r^{3}\right) T_{3}^{3}\right)\right)>0
\end{aligned}
$$

The objective is to determine optimum value of $\mathrm{T}_{3}$ and $\mathrm{T}$ so that $\mathrm{TC}\left(\mathrm{T}_{3}, \mathrm{~T}\right)$ is minimum. The value of $\mathrm{T}_{3}$ and $\mathrm{T}$, for which the total cost $\mathrm{TC}\left(\mathrm{T}_{3}, \mathrm{~T}\right)$ is minimum are the solutions $\frac{\partial T C\left(T_{3}, T\right)}{\partial T_{3}}=0$ and $\frac{\partial T C\left(T_{3}, T\right)}{\partial T}=0$ satisfy the condition $\left\{\left(\frac{\partial^{2} T C\left(T_{3}, T\right)}{\partial T_{3}^{2}}\right)\left(\frac{\partial^{2} T C\left(T_{3}, T\right)}{\partial T^{2}}\right)-\left(\frac{\partial^{2} T C\left(T_{3}, T\right)}{\partial T_{3} \partial T}\right)^{2}\right\}>0$ 


\section{Numerical example}

$\mathrm{P}=25, \beta=0.1, \mathrm{k}=10, \mathrm{r}=0.02, \mathrm{C}_{0}=10, \mathrm{a}=0.6, \mathrm{~b}=0.9, \Upsilon=0.8, \mathrm{C}_{\mathrm{h}}=10, \theta=0.01$

$$
C_{P}=5
$$

Output results are

$$
T_{3}=0.03252714186618942, T=0.4495539743314168
$$

Total cost $=44.55$

\section{Graphical representation of the convexities of $T_{3}$ and $T$ w.r.t T.C}

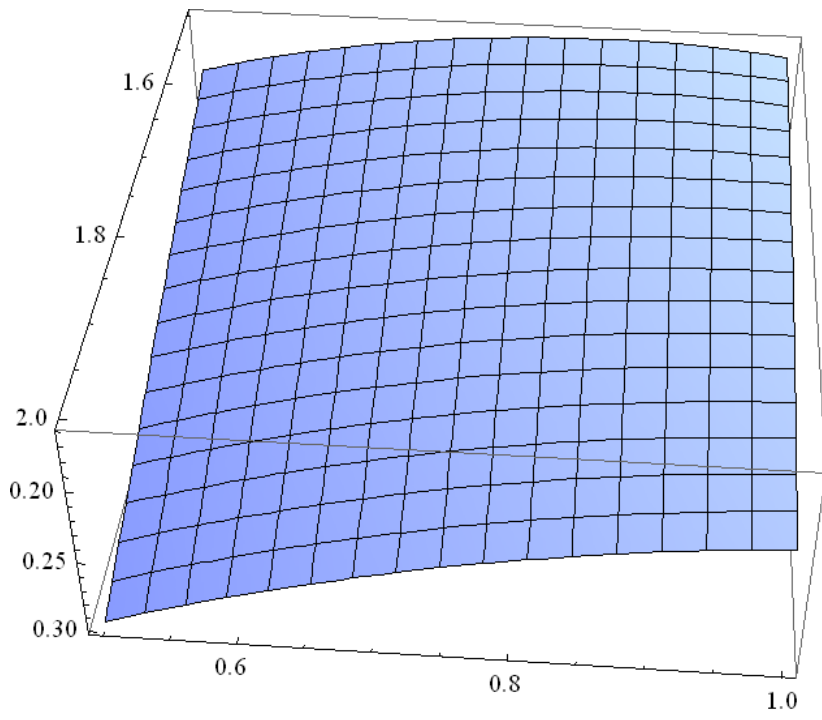

\begin{tabular}{|c|c|c|c|c|c|c|c|c|}
\hline $\begin{array}{c}\text { PARAM } \\
\text { ETER }\end{array}$ & $\begin{array}{c}\text { PARAM } \\
\text { E } \\
\text { TERIC } \\
\text { Value } \\
\end{array}$ & $\mathbf{T}$ & $\mathbf{Q}$ & $\begin{array}{c}\text { PRODUCTI } \\
\text { ON COST }\end{array}$ & $\begin{array}{l}\text { HOLDIN } \\
\text { G COST }\end{array}$ & $\begin{array}{c}\text { ORDERING } \\
\text { COST }\end{array}$ & $\begin{array}{c}\text { DETERIOR } \\
\text { ATION } \\
\text { COST }\end{array}$ & $\begin{array}{l}\text { TOTAL } \\
\text { COST }\end{array}$ \\
\hline \multirow{7}{*}{$\theta$} & 0.01 & 0.449553 & 0.569225 & 2.84612 & 19.368 & 22.2443 & 0.0968402 & 44.5553 \\
\hline & 0.02 & 0.448581 & 0.586937 & 2.93469 & 19.2322 & 22.2925 & 0.192322 & 44.6518 \\
\hline & 0.03 & 0.447618 & 0.604397 & 3.02199 & 19.0987 & 22.3405 & 0.28648 & 44.7476 \\
\hline & 0.04 & 0.447667 & 0.62161 & 3.10805 & 18.9673 & 22.388 & 0.379347 & 44.8428 \\
\hline & 0.05 & 0.445725 & 0.638579 & 3.1929 & 18.8381 & 22.4353 & 0.470953 & 44.9373 \\
\hline & 0.06 & 0.44479 & 0.65531 & 3.2765 & 18.711 & 22.4823 & 0.56133 & 45.0312 \\
\hline & 0.07 & 0.44387 & 0.671808 & 3.35904 & 18.586 & 22.5289 & 0.650508 & 45.1244 \\
\hline
\end{tabular}

TABLE-I

\begin{tabular}{|c|c|c|c|c|c|c|c|c|c|c|}
\hline $\begin{array}{l}\text { PARA } \\
\text { M } \\
\text { ETERS }\end{array}$ & $\begin{array}{l}\text { PARAMETR } \\
\text { IC VALUES }\end{array}$ & $T$ & $\mathbf{Q}$ & $T_{1}$ & $T_{2}$ & $T_{3}$ & $\overline{Q_{1}}$ & $Q_{2}$ & $Q_{3}$ & $\begin{array}{l}\text { TOTAL } \\
\text { COST }\end{array}$ \\
\hline \multirow{4}{*}{$\theta$} & 0.01 & 0.449 & 0.579 & 0.0032 & 0.0260 & 0.0325 & 0.048 & 0.13 & 0.406 & 44.55 \\
\hline & 0.02 & 0.448 & 0.590 & 0.0033 & 0.0268 & 0.0335 & 0.050 & 0.134 & 0.411 & 44.65 \\
\hline & 0.03 & 0.447 & 0.624 & 0.0034 & 0.0276 & 0.0345 & 0.051 & 0.138 & 0.431 & 44.74 \\
\hline & 0.04 & 0.476 & 0.632 & 0.0035 & 0.0284 & 0.0355 & 0.053 & 0.142 & 0.440 & 44.84 \\
\hline \multirow{4}{*}{$C_{P}$} & 2 & 0.5135 & 3.2794 & 0.018739 & 0.14491 & 0.1873 & 0.281 & 0.649 & 2.342 & 39.0122 \\
\hline & 3 & 0.4795 & 2.241 & 0.0128 & 0.1024 & 0.1280 & 0.192 & 0.500 & 1.600 & 41.7678 \\
\hline & 4 & 0.4596 & 1.364 & 0.0077 & 0.0623 & 0.0779 & 0.116 & 0.31 & 0.973 & 43.5758 \\
\hline & 5 & 0.4490 & 0.569 & 0.0032 & 0.026 & 0.0325 & 0.048 & 0.13 & 0.406 & 44.555 \\
\hline \multirow[b]{2}{*}{$\mathrm{C}_{\mathrm{h}}$} & 10 & 0.449 & 0.569 & 0.0032 & 0.026 & 0.0325 & 0.048 & 0.13 & 0.406 & 44.555 \\
\hline & 15 & 0.384 & 1.581 & 0.00902 & 0.0722 & 0.09029 & 0.135 & 0.36 & 1.110 & 52.0276 \\
\hline
\end{tabular}

Table II 


\begin{tabular}{|c|c|c|c|c|c|c|c|c|c|c|}
\hline & 20 & 0.349 & 1.93 & 0.0110 & 0.088 & 0.1104 & 0.165 & 0.44 & 1.38 & 76.467 \\
\hline & 25 & 0.324 & 2.27 & 0.0118 & 0.094 & 0.1184 & 0.177 & 0.47 & 1.48 & 95.67 \\
\hline \multirow{4}{*}{$\mathrm{C}_{\mathrm{O}}$} & 10 & 0.449 & 0.569 & 0.0032 & 0.026 & 0.0325 & 0.048 & 0.13 & 0.406 & 44.555 \\
\hline & 15 & 0.550 & 0.696 & 0.0039 & 0.0318 & 0.0398 & 0.059 & 0.159 & 0.497 & 54.55 \\
\hline & 20 & 0.636 & 0.804 & 0.0045 & 0.0368 & 0.0459 & 0.068 & 0.183 & 0.574 & 62.97 \\
\hline & 25 & 0.712 & 0.902 & 0.0051 & 0.0411 & 0.0513 & 0.076 & 0.200 & 0.640 & 70.38 \\
\hline \multirow{4}{*}{$b$} & 0.6 & 0.4537 & 0.975 & 0.00609 & 0.0487 & 0.0609 & 0.0914 & 0.243 & 0.414 & 44.14 \\
\hline & 0.7 & 0.4519 & 0.831 & 0.00503 & 0.0402 & 0.0503 & 0.0755 & 0.201 & 0.411 & 44.31 \\
\hline & 0.8 & 0.4506 & 0.696 & 0.00402 & 0.0327 & 0.0409 & 0.0614 & 0.163 & 0.409 & 44.45 \\
\hline & 0.9 & 0.449 & 0.569 & 0.00325 & 0.0261 & 0.0325 & 0.0487 & 0.130 & 0.406 & 44.55 \\
\hline \multirow{3}{*}{$\mathrm{r}$} & 0.01 & 0.448 & 0.5695 & 0.003254 & 0.0262 & 0.03254 & 0.04881 & 0.1302 & 0.4070 & 44.58 \\
\hline & 0.02 & 0.449 & 0.5692 & 0.003252 & 0.0261 & 0.03252 & 0.04878 & 0.1301 & 0.4065 & 44.55 \\
\hline & 0.03 & 0.450 & 0.5688 & 0.003250 & 0.0260 & 0.03250 & 0.04875 & 0.1300 & 0.4063 & 44.52 \\
\hline
\end{tabular}

\section{Observations:}

From table 1 with the increase in deteriorate rate, the cyclic time and holding cost decrease but ordering cost, deterioration cost, production cost, total cost and total production quantity increase.

\section{From table 2}

1. With the increase in rate of deteriorating items, total cost, optimum total production quantity, Cycles times $\left(T_{i}, \mathrm{i}=1,2,3\right)$ and maximum inventory increase but cycle time $\mathrm{T}$ decreases.

2. With the increase in setup cost per unit, optimum quantity $\left(\mathrm{Q}^{*}\right)$, maximum inventory and cycle times and total cost increase.

3. With the increase in holding cost per unit, optimum total production quantity $\left(\mathrm{Q}^{*}\right)$, maximum inventory and cycle times $\left(\left(T_{i}, \mathrm{i}=1,2,3\right)\right.$, total cost increase but cycle time $\mathrm{T}$ decrease.

4. With the increase in production cost optimum total production quantity $\left(\mathrm{Q}^{*}\right)$, maximum inventory, cycle times $\left(\left(T_{i}, \mathrm{i}=1,2,3\right)\right.$, cycle time $\mathrm{T}$ decrease but total cost increases.

5. With the increase in inflation rate optimum total production quantity $\left(\mathrm{Q}^{*}\right)$, maximum inventory, cycle times $\left(\left(T_{i}, \mathrm{i}=1,2,3\right)\right.$, total cost decrease \& cycle time $T$ increases.

6. With the increase in parameter $\mathrm{b}$ optimum total production quantity $\left(\mathrm{Q}^{*}\right)$, maximum inventory, cycle times $\left(\left(T_{i}, \mathrm{i}=1,2,3\right)\right.$, cycle time $\mathrm{T}$ decrease $\&$ total cost increases.

\section{Conclusion}

Generally, inventory models are based on the assumption that products generated have indefinitely long lives, but almost all items deteriorate with time. Often, the rate of deterioration is low and there is little need to consider the deterioration in the determination of economic lot size. In this paper, a dynamic inventory model has visited with deteriorating production in which each of the production, the demand and the deterioration rates, as well as all cost parameters are applied to be general functions of time. The objective is to cycle time and optimal production lot size, which minimize total costs. The relevant model is solved by mathematically. Illustrative examples are provided. Our focus in this study is on determining the optimal solution of production time so that total cost of the whole cycle will be minimized.

\section{References}

[1] A.A. Taleizadeh, S.S. Kalantari, L.E. Cardenas-Barron, Determining optimal price, replenishment lot size and number of shipment for an EPQ model with rework and multiple shipments Journal of Industrial and Management Optimization, 11, pp. 1059-1071, 2015.

[2] B. C. Giri, S. Pal, A. Goswami, and K. S. Chaudhuri, An Inventory model for deteriorating items with stock-dependent Demand rate, European Journal of Operational Research, vol. 95, no. 3, 604-610, 1996.

[3] B. Sarkar, L.E. Cardenas-Barron, M. Sarkar, M.L. Singgih, An economic Production quantity model with random defective rate rework process and backorder for a single stage production system Journal of Manufacturing System, 33, 423-435, 2014.

[4] C. Krishnamoorthi, C.K. Sivasankari, Production inventory model for deteriorative items with three levels of production and shortages, Yuguslav Journal of Operations Research, DOI 10.2298/YJOR150630014K, 2016.

[5] C.K. Sivasankari, S. Panayappan, Production inventory model for two - level production with deteriorative items and shortage, International Journal of Advanced Manufacturing Technology 76, 2003-2014, 2014.

[6] C.-Y. Dye and L.-Y. Ouyang, An EOQ model for perishable items under stock-dependent selling rate and time-dependent partial backlogging, European Journal of Operational Research, vol. 163, no. 3, 776-783, 2005.

[7] F. Raafat, Survey of literature on continuously deteriorating inventory model, Journal of the Operational Research Society, 42, 27-37, 1991.

[8] H. K. Alfares, Inventory model with stock-level dependent demand rate and variable holding cost, International Journal of Production Economics, vol. 108, no. 1-2, 259-265, 2007. 
[9] J.A. Buzzacott, Economic order quantities with inflation, Operation Research Quarterly, 26(3) 553-558, 1975.

[10] J.T. Hsu, L.F. Hsu, An integrated vendor-buyer inventory model with imperfect items and planned back orders, International Journal of Advanced Manufacturing Technology, 68(9-12), 2121-2132, 2013.

[11] Jayshree \& S. R. Singh, Analysis of an inventory system with ramp Type demand rate, partial shortages under inflation and learning, Grenze International Journal of Engineering and Technology, Vol 2 (2), 29-40, CrossRef doi 10.21647/gijet/2016/v2/i2/48898, 2016 a.

[12] Jayshree and S. Jain, An EOQ model dealing with Weibull deterioration with shortages, ramp type demand rate and unit production cost incorporating the effect of inflation, Sixth International Conference on Computational Intelligence \& Information TechnologyCIIT-2016, McGraw-Hill Conference Proceeding, 150-163, ISBN-13-9789352604357, 2016.

[13] Jayshree and S.R.Singh, An inventory model for decaying items with ramp type demand under learning effect, Seventh International Conference on Advances in Computing, Control, and Telecommunication Technologies - ACT 2016, 276-283, ISBN-978-81-8487599-7, 2016 b.

[14] K. Karthikeyan, G. Viji Economic production quantity inventory model for three levels of production with deteriorative item, Ain Shams Engineering Journal (in press)

[15] K.-S. Wu,L.-Y. Ouyang, and C.-T. Yang,An optimal replenishment policy for non-instantaneous deteriorating items with stockdependent demand and partial backlogging, International Journal of Production Economics, vol. 101, no. 2, 369- 384, 2006.

[16] L.E. Cardenas-Barron, K.J. Chung, G.Travino-Garza, Celebrating a century of the economic order quantity model in honor of Ford Whitman Harris, International Journal of Production Economics, 155, 1-7, 2014 a.

[17] L.E. Cardenas-Barron, S.S. Sana, A production-inventory model for a two-echelon supply chain when demand is dependent on sales teams' initiatives, International Journal of Production Economics, 155 ,249-258, 2014 b.

[18] R. C. Baker and T. L. Urban, A deterministic inventory system with an inventory-level-dependent demand rate, Journal of the Operational Research Society, vol. 39, no. 9, 823-831, 1988.

[19] R. Gupta and P. Vrat, Inventory model for stock-dependent consumption rate, Opsearch, vol. 23, no. 1, 19-24, 1986.

[20] R. I. Levin, C. P. McLaughlin, R. P. Lamone, and J. F. Kottas, Production/Operations Management: Contemporary Policy for Managing Operating Systems, McGraw-Hill, New York, NY, USA, 1972.

[21] R.B Misra, Optimum production lot-size model for a system with deteriorating inventory, International Journal of Production Research, 13 (5), 495-505, 1975.

[22] S.K. Goyal, B.C. Giri, Recent trends in modeling of deteriorating inventory, European Journal of Operational Research, 134, 1-16, 2001. 